\title{
Healed and Crippled: The Effect of Global Medicine on African Indigenous Treatment and Care Approaches
}

\author{
Emmanuel Mutungi \\ Maseno University, Kisumu County, Kenya \\ Kyambogo University, Kampala, Uganda \\ Felix Ngunzo Kioli \\ Maseno University, Kisumu County, Kenya \\ Benson Azariah Mulemi \\ Catholic University of Eastern Africa, Nairobi, Kenya
}

\begin{abstract}
Whereas global medicine and health care practices have improved the quality of people's lives, especially in the developing countries data abounds that local communities have been crippled by the same medical practises. Some societies in developing countries have become sources of specimen for clinical trials of biomedicine which is unaffordable to their citizens. This paper explores the neglect of traditional African medicinal innovations and research in favour of imported Western medicine perpetuated by the developed countries. The paper argues that global medicine and health care have neither utilized nor recognized the African Traditional Medicine (ATM) fully, despite the fact that cultures in developed world used and continue to utilize the indigenous medical knowledge. The paper further argues that instead of neglecting African Traditional Medicine, ATM and biomedicine can be more beneficial by blending them into a single system, through what we would call in this paper High-Performance Medical Research (HPMR). This would allow participation of communities to achieve both socio-economic and medical knowledge growth rather than being a monopoly and preserve of developed organizations in the North. This paper proposes that HPMR should be a systematic and scientific approach for enhancing local people's participation in the development of medical ventures. This paper draws on secondary data on traditional African therapeutic practices by some local communities in East Africa combined with literature review on medical practice in Western societies.
\end{abstract}

Keywords: global medicine, treatment, care, African traditional Medicine, western medical practices, developing countries

\section{Introduction}

The notion that global medicine which is essentially Western medicine has improved the quality of people's lives worldwide is one of the assumptions imposed on a many cultural communities around the world

Emmanuel Mutungi, Ph.D. Student, Maseno University/Lecturer, Faculty of Vocational Studies, Department of Art and Industrial Design, Kyambogo University.

Felix Ngunzo Kioli, Ph.D., Lecturer, School of Arts \& Social Sciences, Department of Sociology and Anthropology, Maseno University.

Benson Azariah Mulemi, Ph.D., Lecturer, Faculty of Arts and Social Sciences, Department of Social Sciences, Catholic University of Eastern Africa. 
by proponents of Western medicine. Every society is guided by a set of beliefs, values and norms that inform well-being of that particular community and which can only be distorted but not changed by external factors. Waldron (2010, p. 50) observed that:

Every society embraces particular "cultural theories" or ideologies that set the parameters within which normal, abnormal and deviant behaviour is defined. These cultural theories on illness, treatment/healing and health often stem from diverse observations, understandings and interpretations of specific symptoms, the behaviour of persons affected by illness and how symptoms are uniquely experienced and explained in specific cultures.

In Africa, treatment was and remains a community undertaking. Each community has special category of people who are known to be good at treating certain ailments. Expertise knowledge in treating such ailments is based on experience almost in a similar way expertise in western medicine is also based on experience. It is unusual to find a young experienced healer in traditional medicine as it is not common to find a young consultant in the western medicine. Although, with the coming of modern technology this is changing in the contemporary western medicine as we now have young consultants, still the most respected professors with wide knowledge are of age. The young therefore can be consultants but in a specific fields.

Many African communities and especially the poor use indigenous medicine in their lifetime either for cultural reasons or as a result of economic capabilities. Old people have remained first health contacts in many families before a sick person is taken to a health facility. In situations where the family of the sick person does not afford conventional treatment, traditional medicine is always administered to the patient. It is this reason that several governments are trying to find a way of harmonising traditional medicine with the conventional one. Much as this is a good move to promote indigenous medicine, decades of disregard from the Government have created mistrust between the practitioners of conventional medicine and those of traditional medicine (Hillenbrand, 2006).

This paper presents prepositions that will blend the indigenous medicine with the contemporary medicine through a single system we refer to as High-Performance Medical Research (HPMR), which allows participation of communities to achieve both socio-economic and medical knowledge growth by merging both the indigenous knowledge on medicine with the laboratory based conventional medicine. The paper argues that instead of medicine remaining a monopoly and preserve of developed organizations of the western medicine, the two should work together to develop culturally and conventionally accepted medicine.

\section{Methodology}

This paper draws on secondary data on traditional African therapeutic practices by some local communities in East Africa combined with literature review on medical practice in Western societies. In reviewing the literature, our sources were (1) people, (2) articles and bibliographies, and (3) online databases (Bernard, 2006). We talked to different people to establish their perceptions about the impact of western medicine on traditional medicine. We listened to different people who use traditional medicine and why, and those who do not use it. We interacted with the older people who knew some traditional medicine and also who use western medicine. We talked to the young who know much of the contemporary medicine and little of the traditional medicine. We consulted a number of publications that had dealt with the subject and compared them with the current use of both traditional and western medicine. We also benefited from various online databases especially those that informed the study. We triangulated the gathered information and then analyzed the emerging themes for us to draw thematic conclusions. 


\section{The Results}

Analysing how to improve the collaboration between traditional and conventional medicine in Cameroon, Hillenbrand (2006, p. 1) posits that there is a disagreement between promoters of traditional and conventional medicine. That advocates for conventional medicine argue that; traditional medicine is fraught with problems of imprecise dosage, poor diagnosis, charlatanism, exaggerated claims of abilities, and inadequate knowledge of anatomy, hygiene, and disease transmission. Traditional practitioners on the other hand believe that conventional medicine practitioners and research scientists condemn their practice in order to steal their creativity and innovations.

The fact that $80 \%$ of the population in developing countries still use traditional medicine demonstrates that western medicine is not easily accessed by everyone especially those in rural areas. We propose that Africa may benefit from utilizing the knowledge and resources within their reach and later develop it for other communities globally. Govindarajan \& Trimble (2012) call this reverse innovation. Govindarajan and Trimble (2012) explain reverse innovation as a strategy of innovating in emerging (or developing) markets and then distributing/marketing these innovations in developed markets. Why this could be very helpful is the fact that already $80 \%$ of the population are clients of TM and therefore there would not be any need to rely on western medicine supplies. Countries such as China and India have already become big destination of manufacturing companies including pharmaceuticals which products are then supplied globally. The thinking that innovations come from rich countries and sold to poor countries fails to measure up because innovations must be customised to the needs of the users.

\section{Marginalization of African Indigenous Medicine: From Preventive to Curative}

World Health Organization (2000) defines traditional medicine as:

the sum total of the knowledge, skills and practices based on the theories, beliefs and experiences indigenous to different cultures, whether explicable or not, used in the maintenance of health, as well as in the prevention, diagnosis, improvement or treatment of physical and mental illnesses. (p. 1)

Traditional medicine is a practice that is as old as the society because before the discovery of the scientific alternative medicine, society depended on traditional medicine largely got from the environment. Some cultures in India and China have developed more detailed systems of traditional medicine which they use for different ailments. Because traditional medicine was got from the environment, it is cheap, readily available and affordable to many communities. The knowledge of using it is passed from the older generation to the young hence in any community there is a lineage of practitioners of traditional medicine.

Traditional knowledge related to health and treatment of various ailments is found in many communities around the world. In Africa, use of plant based traditional medicine is on the increase as the population increases (Rukangira, n.d.). Essentially in the developing countries, traditional medicine forms the primary mode of health care that covers $80 \%$ of the population (World Health Organization, 2008). Moreover, research reveals that traditional and scientific medicine are always used to complement one another. The scientific medicine is used to suppress the symptoms while traditional medicine helps in restoring the body to its natural balance (Soh \& Walter, 2009). Consequently, the 50th session of World Health Organization (WHO) Regional committee for Africa officially recognized that use of traditional medicine has the potential of availing each member of the community appropriate medication which will enable Africa to achieve health 
for all (Sambo, 2010).

The committee hence recommended accelerating development and research in traditional medicine to improve access of health care to the majority of the population. However, to effectively revive the use of traditional medicine requires the involvement of the community because communities know which plants have the medicinal components and the habitat of each plant. Unfortunately, a century or so of neglecting traditional medicine means that some of the practices have been forgotten. Noticing that gap, the Centre for Disease Control and Prevention (CDC) advocates for community engagement so that community can work hand in hand with the alternative medicine promoters to unveil some of the medicinal plants and other therapeutic practices. Centre for Disease Control and Prevention [CDC] (2011, p. 7) defines community engagement as "the process of working collaboratively with and through groups of people affiliated by geographic proximity, special interest, or similar situations to address issues affecting the well-being of those people". Furthermore, CDC defines community as a group of people united by a common characteristic such as shared interests, values or experiences or traditions. For any initiative to take root in a community, members need to understand the purpose and to own it with an anticipation to benefit from the outcome. Indeed, this analysis of CDC shows how ATM has been side-lined and having noticed that western medicine is not reaching every member of the community, traditional medicine is now promoted.

Research further shows that indigenous knowledge (IK) associated with traditional medicine (TM) still exist and are appreciated by many communities. For example, in Uganda, many people use TM as the first line of health care in case one falls sick (Tabuti, Kukunda, Kaweesi, \& Kasilo, 2012). We can ably argue that the failure of the alternative medicine to reach every member of the community has necessitated the increased use of ATM in communities because whereas the ATM was easily available in the community and in the environment, the western medicine is laboratory based, imported and has to be paid for before administering it to a sick person. The traditional practice was that a sick person or the caretakers would pay a small fee commonly known as Obuteerakishaka in Runyankore or Ekikubansiko in Luganda. This was not necessarily paid in cash it could be a chicken or goat and once one was healed then payment was made. This is not a case with western medicine where one has to pay consultation fee to meet a health worker to explain the sickness. On the other hand governments do not have the capacity to provide free medical services to every member of the community. As a result there are no drugs in public health facilities and community members do not have adequate resources to visit expensive hospitals which are expensive and most of the time far away. As a result the community resort to traditional medicine. It is estimated that for example $90 \%$ of the population in Ethiopia, $70 \%$ in Rwanda, and 60\% in Uganda and Tanzania use traditional medicine for their primary health care (Tabuti et al., 2012). In Uganda, traditional medicine is widely used for the prevention, diagnosis and treatment of social, mental and physical illness.

Whereas contemporary western medicine regards traditional medicine as non-scientific, research shows that indigenous forms of healing were carefully studied and selected by the users. Akomolafe (2012, p. 728) posit that "indigenous forms of healing are culture-sensitive, holistic, spiritual, collectivistic, and rich in variety". Akomolafe further refers to Solberg, Ritsma, Davis, Tata, and Jolly (1994) and Garrett and Wilbur (1999) who observe that Asians sustain close-knit family networks that serve as healing sources in times of distress while some American Indians engage in elaborate sun-dances and pipe festivals in their quest for a sustained sense of rejuvenation and wellbeing. Unfortunately, promoters of western medicine continue to portray a negative perception on indigenous medicines as Waldron (2010) and Adefolaju (2014) state that 
health systems and perspectives different from Euro-Western paradigms are marginalized and rendered illegitimate, and in many cases equated to fetishes and the practitioners derided and tagged "witch-doctors". Waldron (2010, p. 50) while examining the production of knowledge in the health systems in Western and non-Western societies goes on to argue that scientific knowledge in Western medicine serves to foster and sustain the marginalization of "African indigenous" health knowledge, that the epistemological terrain upon which both indigenous and Western health professionals traverse is not level, resulting in a hierarchy of knowledge, as well as superficial dichotomies between the indigenous and Western health approaches that obscure opportunities for alliances at the epistemological crossroads.

\section{Rejection of African Medicine and Medical Knowledge}

When western medicine and colonial administration were introduced in Africa in late 19th century by missionaries, they affected the indigenous medicine, creativity and innovation were put to sleep (Mutungi, 2013), and traditional medicine was thrown to the background while African researchers and innovators became "witch-doctors" (Adefolaju, 2014). This was because the Christian teaching considered African practices, material culture and healing therapies unchristian and therefore evil. This marginalization of African healing practices and innovation is still going on as Adefolaju (2014, p. 121) puts it that "rejection and derision of the indigenous healing practice has continued till present time, leading to government's unwillingness to recognise and harmonise it with the global system of health care delivery".

Why did western medicine reject Africa's long practiced forms of healing? Why didn't African resist and continued with their forms of healing practices. We may never find answers to these questions but what is obvious is that the proponents of western medicine used their religion-Christianity and technology which to them was advanced and influenced the indigenous perception of their medicine and appreciation of what was African. With such strategic approach, the African mind was greatly affected by the new thinking and it was occupied by the western ideology. To illustrate this the author borrow the interpretation of the mind by Harris $(2009$, p. 7) who considers the mind as neither a friend nor enemy. In his interpretation of the mind he observes that the mind is a double-edged sword.

It's very useful for all sorts of purposes, but if we don't learn how to handle it effectively, it will hurt us. On the bright side, language helps us make maps and models of the world; predict and plan for the future; share knowledge; learn from the past; imagine things that have never existed and go on to create them; develop rules that guide our behavior effectively and help us to thrive as a community; communicate with people who are far away; and learn from people who are no longer alive.

Whereas the western used the mind to cripple the African creativity, they used the same mind to promote their cultures, beliefs, and innovations. In Africa, once the western thinking was introduced, creative innovations, research and production were put to sleep and Africans prescribed to western made policies, innovations, forms of governance, and treatment without questioning. Contrary, the western ideologies used their mind to promote research and innovation of new products which were then sold to Africans. What Africans were encouraged to do was to produce raw materials to supply the industries located in western economies. They created new models of the world in health, in economics and politics which are exported to many countries especially the developing countries. Whereas the Harris talks of predicting the future basing on the past, African past was labelled evil and the African mind does not want to associate with it. In other words, this makes indigenous communities dependant on western thinking including medicine. 
Indigenous African medicine was both preventive and curative. The African medicine does not wait to carry out laboratory tests but rather it emphasises prevention. Ethnography in some cultures of Uganda and East Africa reveal that society had certain things that members had to observe for their well-being. For example many tribes in Uganda and Kenya carry out male circumcision. This is practice that societies have been practicing for a long time much earlier than the coming of Muslims who circumcise. Every male adult had to go through it and it signified maturity and was a process of initiating one into adulthood. Coming back to this paper, this could be assigned another meaning - maybe it was to reduce on sexually transmitted diseases since one become an adult after circumcision. This argument is based on the fact that western medicine recently fronts circumcision as a measure of preventing the spread of HIV/AIDS. The argument here is that it should not have taken this long for proponents of western medicine to understand that circumcision was helpful in reducing sexually transmitted infections (STI). Circumcision did not only stop with men but also it was practiced in women. Research reports 28 African countries and other several countries elsewhere to be practicing it. Communities in Eastern Uganda and Western Kenya practice it although it has become infamous and now referred to as female genital mutilation (FGM). This paper does not in any way promote FGM but it factors in the therapeutic values that could be enshrined in the act. Although the World Health Organization regards it as simply for cultural and non-therapeutic reasons, ethnography in such communities reveals that it was indeed therapeutic. It is argued that men subjected women to this act to reduce their libido and to prolong their orgasm. Although this is not a reason good enough to support it because it violates the rights of women, it does not take away the fact that it was therapeutic. What the western thinking could have done was to be mindful of why it was practiced and come up with remedies that were accepted to the community. In that way it should not have become a secret affair as it is the case today.

When we talk of the proponents of western ideologies to have been mindful, we believe that had they been mindful and integrated their medicine with the TM, the communities would have owned the medicines. Moreover, mindfulness means paying attention with flexibility, openness, and curiosity (Harris, 2009). The western medicine ignored the attributes of mindfulness. They were not flexible to understand why indigenous people believed and used the medication the way they did, they were not open to the indigenous people to explain how best the indigenous medicine could be improved; instead TM was taken as fetish and devilish, and they were never curious before making conclusions about TM. Mindfulness as a concept is as old as human race. It was because of mindfulness that man discovered fire because he wanted to warm himself and to cook food, it was mindfulness that tools were discovered, it was mindfulness that communities kept together. Ethnography from one of the tribes in Uganda reveals that for that community to discourage intruders in marriage, they trained the young people before marriage that a man had to give a sharp knife to the wife whenever they were to play sex. The woman would then hold the knife pointing to the man during the exercise but not to harm the man. One of the stories goes that a man from a different tribe convinced a young woman to have an affair with him. The woman agreed and the two met at their agreed place. After making themselves comfortable, the woman reached for her bag pulled out a sharp knife ready to receive the man as was the practice in her culture. On seeing the knife, the man took off and run with his clothes in his hands. We argue that this was a therapeutically planed act by senior members of the community to keep away men from other cultures from going out with their women.

It evidently emerged that despite the fact that western medicine has been used for over a century on African continent and other developing nations, traditional medicine has remained the first line of treatment to 
more than $80 \%$ of the population in developing countries. This revelation becomes a trajectory on which to gauge the relevancy of traditional medicine in the 21 st century. The failure of western prescribed policies to cover the entire population in developing countries resulted in communities resorting to the use of traditional medicine in which they had skills and experience for centuries. Use of western medicine has remained expensive more especially to the majority of ordinary communities in rural areas. The medicine is manufactured by commercial firms who want to maximize profits contrary to the traditional medicine which was a responsibility of elders and for the good of the community. In addition, western medicine facilities are located far away from the communities. In Uganda for example, government has tried to establish different health facilities at different administrative levels. These are village health educators responsible to smaller villages, Health Centre II at parish level, Health Centre III and sub-county, Health Centre IV at county level and the referral hospital at the districts. However, despite this beautiful establishment most of these facilities either lack drugs or manpower. Communities therefore, resort to using traditional medicine which they are able to access within their environment.

\section{High-Performance Medical Research (HPMR) for African Traditional Medicine and Western medicine}

Waldron (2010) examined various examples of successful collaborative models that attempt to bridge the gap between indigenous and Western health practices. Models that also give a generational evolution and flexibility among different cultural groups whose access to health services fall between western and indigenous healing practices. Such communities can use western medicine if they can afford, but also can rely on traditional medicine if they cannot afford or as the first line of treatment before they consult western medicine. In most developing communities worldwide, this approach of treatment is employed. Many families administer traditional medicine alongside western medicine. The ethnography from families in Uganda indicated that parents are discouraged to engage in any sexual activity or prepare meat in a home if any of their children is suffering from measles. The explanation of this practice came to the conclusion that patients suffering from measles need a lot of care therefore the remedy is meant discourage any pleasures in a home and to allow focus on the patient. In addition there are several traditional medicine that are given to measles patients alongside the western medicine. Most of these are nutritious foods which boost the immunity of the patient.

The collaboration therefore brings in discipline restraining family members from excessive pleasure, nutritious feeding of patients and western curative drugs. We need interface the two approaches to treatment to achieve high performance in treatment of these ailments. We need to be mindful of people's perceptions so as to help them become resilient and manage their healthcare issues. Mindfulness requires flexibility, openness, and curiosity. So long as the communities realize that their beliefs are not listened to, that the western medicine under looks their traditional approaches to health issues, they are likely to go secret. When communities feel despised and rejected because of the way they approach a healthy problem, they will not open up and the practice will continue at the back of a public eye. This is what has happened with most traditional practices in many societies especially when Christianity called indigenous medicine ungodly. As a result some families developed a double sided approach of publically using modern medicine and applying indigenous medicine in secrete. 


\section{Conclusion}

Openness is an attribute that will bring in high performance medical approaches. Promoters of western medicine need to be open to the usefulness of traditional medical practices, material medical and therapies such that TM medicine is not secretly practiced. Governments also need to accept that western medicine cannot be accessed by every person. They need to promote research in use of medical practices that are based on indigenous knowledge that is not expansive to access. Both promoters of western medicine and governments need to be curious of the fact that people continue to use TM even when western medicine is given out free or at concessional prices. They need to interrogate why TM remains the first line of treatment to many families before they approach a contemporary health worker of facility. It becomes apparent therefore, that western medicine cannot totally wipe out traditional medicine because TM is more than mere curative but is also largely preventive.

Payyappallimana (2006) observed that there has an ongoing growing interest in traditional medicine and its relevance to public health both in developed and developing countries. That a number of factors such as "diversity, flexibility, easy accessibility, broad continuing acceptance in developing countries and increasing popularity in developed countries, relative low cost, low levels of technological input, relative low side effects and growing economic importance" underlie continued use of traditional medicine quoted World Health Organization (WHO) in 2002. It is therefore important to mainstream and align traditional medicine with western medicine into public health care to enable communities to access treatment they understand and can afford. Governments need to acknowledge that worries WHO worry about such safety, efficacy, quality and rational use of traditional medicine should not hamper the use of traditional medicine. Traditional Medicine and biomedicine need be blended into a single High-Performance Medical Research (HPMR) system, which allows participation of communities to achieve both socio-economic and medical knowledge growth rather than being a monopoly and preserve of developed organizations in the North.

\section{References}

Adefolaju, T. (2014). Traditional and orthodox medical systems in Nigeria: The imperative of a synthesis. American Journal of Health Research, 2(4), 118-124.

Akomolafe, A. C. (2012). Decolonizing the notion of mental illness and healing in Nigeria, West Africa. Journal of Critical Psychology in Changing World (pp. 726-740).

Bernard, H. R. (2006). Research methods in anthropology: Qualitative and quantitative approches. UK: Altamira Press.

Centre for Disease Control and Prevention (CDC). (2011). Principles of community engagement (2nd ed.). Retrieved from http://www.atsdr.cdc.gov/communityengagement/pdf/PCE_R port_508_FINAL.pdf

Harris, R. (2009). ACT made simple: An easy-to-read primer on acceptance and commitment therapy (1th ed.). Oakland, CA: New Harbinger Publications.

Hillenbrand, E. (2006). Improving traditional-conventional medicine collaboration: Perspectives from Cameroonian traditional practitioners. Nordic Journal of African Studies, 15(1), 1-15.

Martha, A. W. (n.d.). Community engagement community viability specialist virginia cooperative extension. Retrieved from http://walker53@vt.edu

Mutungi, E. (2013). When the butter got done: The resilience of indigenous design processes during dictatorial regime in Uganda between 1971-1978 and their continued use in Kiruhura district in S.W. Uganda. Proceedings from Gaborone International Design Conference, Gaborone Botswana.

Payyappallimana, U. (2006). Role of traditional medicine in primary health care: An overview of perspectives and challenges. Yokohama Journal of Social Sciences, 14(6), 57-77.

Rukangira, E. (n.d.). Medicinal plants and traditional medicine in Africa: Constraints and challenges. Retrieved from http://infohouse.p2ric.org/ref/40/39776.pdf 
Sambo, L. G. (2010). The decade of African traditional medicine: Progress so far. Retrieved from http://ahm.afro.who.int/issue13/pdf/AHM\%2013\%20Special\%20Issue\%20Pages\%204to6.pdf

Soh, N. L., \& Walter, G. (2012). Traditional and alternative medicine treatments in child and adolescent mental health. Retrieved from http://iacapap.org/wp-content/uploads/J.2-ALTERNATIVE-072012.pdf

Tabuti, J. R. S., Kukunda, C. B., Kaweesi, D., \& Kasilo, O. M. J. (2012). Herbal medicine use in the districts of Nakapiripirit, Pallisa, Kanungu, and Mukono in Uganda. Journal of Ethnobiology and Ethnomedicine, 8(35), 1-15.

Waldron, I. (2010). The marginalization of African indigenous healing traditions within western medicine: Reconciling Ideological tensions \& contradictions along the "Epistemological Terrain". Women's Health and Urban Life, 9(1), 50-68.

World Health Organization. (2008). Fact Sheet No 134 Traditional medicine (updated) Dec 2008. 